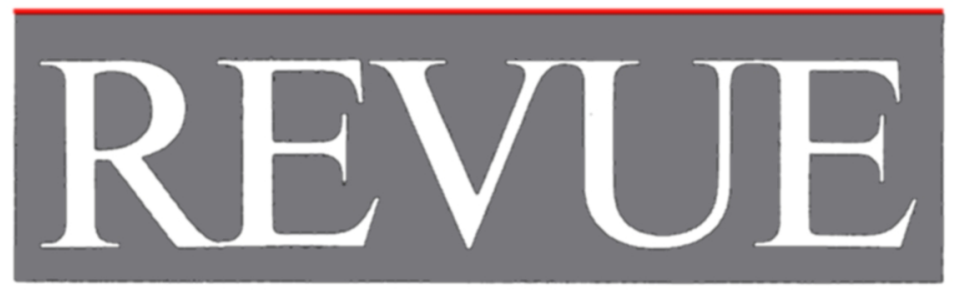

\title{
INTERNATIONALE
}

\section{DE LA CROIX-ROUGE}

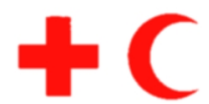

Publication bimestrielle du Comité international de la Croix-Rouge

au service du Mouvement international de la Croix-Rouge et du Croissant-Rouge

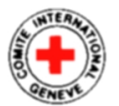




\section{COMITÉ INTERNATIONAL DE LA CROIX-ROUGE}

M. CORNELIO SOMMARUGA, docteur en droit de l'Université de Zurich, $\mathrm{d}^{r}$ h.c. des Universités de Fribourg (Suisse), do Minho, Braga (Portugal), de Bologne (Italie), de NiceSophia Antipolis (France) et de Séoul (République de Corée), président (membre depuis 1986)

MM. PIERRE KELLER, docteur en philosophie en relations internationales (Yale), banquier, viceprésident (1984)

ERIC ROETHLISBERGER, docteur en sciences politiques de l'Institut universitaire de hautes études internationales de Genève, vice-président (1994)

ULRICH GAUDENZ MIDDENDORP, docteur en médecine, privat-docent de la faculté de médecine de l'Université de Zurich, ancien chef de la clinique chirurgicale de l'Hôpital cantonal, Winterthour (1973)

$M^{\text {me }}$ RENÉE GUISAN, secrétaire générale de l' «Institut de la Vie international», directrice d’établissements médico-sociaux vaudois, membre de l'«International Association for Volunteer Effort» (1986)

$\mathrm{M}^{\mathrm{me}} \quad$ ANNE PETITPIERRE, docteur en droit, avocate, professeure à la faculté de droit de l'Université de Genève (1987)

M. PAOLO BERNASCONI, avocat, lic. iur., chargé de cours de droit fiscal et de droit pénal économique aux Universités de Saint-Gall, de Zurich et de Milan (Bocconi), ancien Procureur Général à Lugano

$\mathrm{M}^{\text {me }} \quad$ LISELOTTE KRAUS-GURNY, docteur en droit de l'Université de Zurich (1988)

$M^{\text {me }} \quad$ SUSY BRUSCHWEILER, infirmière, ancienne directrice de l'Ecole supérieure d'enseignement infirmier de la Croix-Rouge suisse à Aarau, directrice générale SV-Service, restauration de collectivités (1988)

MM. JACQUES FORSTER, docteur en sciences économiques, professeur à l'Institut universitaire d'études du développement (IUED) à Genève (1988)

JACQUES MOREILLON, licencié en droit, docteur ès sciences politiques, secrétaire général de l'Organisation mondiale du Mouvement Scout, ancien directeur général au CICR (1988)

MAX DAETWYLER, licencié en sciences économiques et sociales de l'Université de Genève, «Scholar in Residence», International Management Institute (IMI) de Genève (1989)

RODOLPHE DE HALLER, docteur en médecine, privat-docent de la faculté de médecine de l'Université de Genève, président de l'Association suisse contre la tuberculose et les maladies pulmonaires (1991)

DANIEL THÜRER, docteur en droit, LL.M. (Cambridge) professeur ordinaire à l'Université de Zurich (1991)

$M^{\text {me }} \quad$ FRANCESCA POMETTA, licenciée ès lettres, ancien Ambassadeur de Suisse (1991)

MM. JEAN-FRANÇOIS AUBERT, docteur en droit, professeur à l'Université de Neuchâtel, ancien député au Conseil national et au Conseil des Etats (1993)

JOSEF FELDMANN, docteur ès lettres, professeur titulaire de l'Université de Saint-Gall, commandant de corps d'armée à disposition (1993)

M $^{\text {me }}$ LILIAN UCHTENHAGEN, docteur en sciences économiques de l'Université de Bâle, ancienne députée au Conseil national (1993)

M. GEORGES-ANDRÉ CUENDET, licencié en droit de l'Université de Genève, diplômé de l'Institut d'études politiques de l'Université de Paris, M.A. de l'Université de Stanford (USA), membre du Conseil administratif de Cologny/GE (1993)

M. ERNST A. BRUGGER, docteur en sciences naturelles, directeur de FUNDES (Fondation pour la promotion et le développement durable de petites entreprises), conseiller économique, professeur titulaire de l'Université de Zurich (1995)

\section{CONSEIL EXÉCUTIF}

\section{CORNELIO SOMMARUGA, président}

M. ERIC ROETHLISBERGER, vice-président permanent M. PETER FUCHS, directeur général M. JACQUES FORSTER, membre du CICR M. JEAN DE COURTEN, directeur des Opérations $M^{\mathrm{mc}}$ ANNE PETITPIERRE, membre $d u$ CICR $\quad$ M. YVES SANDOZ, directeur de la Doctrine, du Droit et des Relations avec le Mouvement

Le Comité international de la Croix-Rouge (CICR) et la Fédération internationale des Sociétés de la Croix-Rouge et du Croissant-Rouge forment, avec les Sociétés nationales de la Croix-Rouge et du Croissant-Rouge, le Mouvement international de la Croix-Rouge et du Croissant-Rouge.

Institution humanitaire indépendante, le CICR est à l'origine du Mouvement. Intermédiaire neutre en cas de conflits armés et de troubles, il s'efforce d'assurer, de sa propre initiative ou en se fondant sur les Conventions de Genève, protection et assistance aux victimes des conflits armés internationaux et non internationaux et des troubles et tensions internes. 


\section{REVUE INTERNATIONALE DE LA CROIX-ROUGE}

\section{SOMMAIRE}

MAI-JUIN 1996

$N^{\circ} 819$

Éditorial

Le nouveau Protocole sur les armes à laser aveuglantes par Louise Doswald-Beck

Un humaniste des temps modernes: Fiodor Fiodorovitch Martens (1845-1909)

par Vladimir Poustogarov

L'activité humanitaire du mouvement espérantiste pendant les deux guerres mondiales et son rapport avec la Croix-Rouge internationale par José María Rodríguez Hernández

\section{COMITÉ INTERNATIONAL DE LA CROIX-ROUGE}

Trois délégués du CICR tués au Burundi

Face aux défis d'aujourd'hui et de demain, le Mouvement international de la Croix-Rouge et du Croissant-Rouge doit-il repenser son éthique? par Eric Roethlisberger.

Les délégations régionales du Comité international de la Croix-Rouge par Jean-Luc Blondel

Les services consultatifs du CICR en droit international humanitaire: le défi de la mise en œuvre sur le plan national par Paul Berman.

Atelier national sur la mise en œuvre du droit international humanitaire en Namibie (Midgard Resort, 21-23 février 1996).

De retour au Libéria, le CICR en appelle à une réflexion de fond

Communiqué de presse du CICR du 22 avril 1996.

Libéria, la logistique humanitaire en question

par Jean-Daniel Tauxe 
Respect des populations civiles au Liban et dans le nord d'Israël: appel du CICR

Communiqué de presse du CICR du 16 avril 1996

\section{DANS LE MONDE DE LA CROIX-ROUGE ET DU CROISSANT-ROUGE}

Regard sur la Croix-Rouge du Viet Nam

par Dr. Nguyen Van Noi

Mali: les femmes et la guerre

\section{FAITS ET DOCUMENTS}

Troisième session de la Conférence d'examen des États parties à la Convention des Nations Unies de 1980 sur certaines armes classiques - Genève, 22 avril-3 mai 1996

par Peter Herby

Protocole sur l'interdiction ou la limitation de l'emploi des mines, pièges et autres dispositifs, tel qu'il a été modifié le 3 mai 1996 (Protocole II, tel qu'il a été modifié le 3 mai 1996)

Bosnie-Herzégovine: rechercher les disparus par Christophe Girod

Adhésion aux Protocoles additionnels du Commonwealth de la Dominique

République de Colombie: déclaration selon l'article 90 du Protocole I.

\section{LIVRES ET REVUES}

Claude Emanuelli, Les actions militaires de l'ONU et le droit international humanitaire, par Georges P. Politakis

A.P.V. Rogers, Law on the Battlefield par Bruno Doppler

Rony Braumann, Le dilemme humanitaire. Entretien avec Philippe Petit par Jean-François Berger

Publications récentes

\section{DIVERS}

Prix Paul Reuter

DIH CD-ROM 